\title{
CLASSIFICAÇÃO DOS PESTICIDAS USADOS NA BACIA HIDROGRÁFICA DO ITAJ AÍ (SC) QUANTO AO RISCO DE DEGRADAÇÃO DOS RECURSOS HÍDRICOS
}

ADILSON PINHEIRO *

FABIANADE CARVALHO ROSA **

Este trabalho teve por objetivo estimar o risco de degradação das águas superficiais e subterrâneas da bacia do rio Itajaí, mais especificamente na região do Alto Vale do Itajaí, ocasionado pela utilização de pesticidas pelos produtores rurais. O estudo envolveu o levantamento das culturas desenvolvidas na região e suas respectivas áreas, levantamento dos produtos comerciais utilizados e suas características e, a avaliação de risco de degradação das águas superficiais e subterrâneas (método SIRIS) da parte superior da bacia do rio Itajaí. Constatou-se que são utilizados cerca de 39 ingredientes ativos de herbicidas, 33 de inseticidas e 32 de fungicidas nos cultivos de milho, cebola, fumo, arroz, feijão e pastagem. Na cultura de cebola são utilizados cerca de 50 ingredientes ativos de pesticidas. Segundo o método SIRIS, poucos ingredientes ativos foram classificados com risco alto ou muito alto de degradação das águas superficiais e de águas subterrâneas. O herbicida Diurom e o inseticida Carbofurano são os ingredientes ativos com risco muito alto de degradação das águas superficiais.

PALAVRAS-CHAVE: AVALIAÇÃO DE RISCO AMBIENTAL; PESTICIDAS; ÁGUAS SUBTERRÂNEAS; ÁGUAS SUPERFICIAIS; ALTO VALE DO ITAJAÍ.

* Professor, Doutor em Física e Química Ambiental, Departamento de Engenharia Civil, Universidade Regional de Blumenau (FURB), Blumenau, SC (e-mail: pinheiro@furb.br).

** Bióloga, Mestre em Engenharia Ambiental pela FURB, Blumenau, SC (e-mail: fa_rosa@hotmail.com). 


\section{INTRODUÇÃO}

A utilização de pesticidas tem aumentado nas últimas décadas e contribuído para o rendimento da colheita e o decréscimo nos custos de produção (BOWMER et al., 1998). As funções básicas dos pesticidas na agricultura incluem a elevação da produção com aumento da produtividade, a melhoria da qualidade dos produtos e a redução do trabalho e dos gastos com energia. No entanto, o uso de pesticidas tem provocado problemas ambientais e à saúde humana (KOLPIN et al., 2000; BATTAGLIN et al., 2003; SCHULZ, 2004; COUTINHO et al., 2005).

O Brasil, desde a década de 70, destaca-se como um dos maiores consumidores mundiais de pesticidas. Cerca de 670 marcas comerciais são vendidas no Brasil, sendo que $56 \%$ dos produtos registrados são considerados como genéricos. Em 2004 foram comercializados cerca de 230 mil toneladas de pesticidas com faturamento da ordem de US\$4.500 milhões (SINDAG, 2006).

Pesquisa realizada pelo IBGE (2005) indicou que cerca de 1150 municípios brasileiros apresentavam degradação do solo por fertilizantes e pesticidas. Santa Catarina foi apontado como 0 estado com maior proporção de contaminação, uma vez que $56 \%$ dos seus municípios reportaram a ocorrência de contaminação no solo por pesticidas. Esse é o caso da bacia do Itajaí, situada na vertente Atlântica do Estado de Santa Catarina, em especial, na região do Alto Vale. A agricultura representa importante parcela da economia regional e pesticidas têm sido utilizados visando aumentar a produtividade agrícola das culturas.

A EPAGRI (2001) efetuou levantamento da estrutura fundiária, da condição agrária dos produtores rurais, da distribuição do uso das terras agrícolas e do uso dos principais pesticidas na região do Alto Vale do Itajaí. Foi constatada a predominância de pequenas propriedades (91,1\% com menos de 50 ha), abrangendo cerca de $63,6 \%$ da área total. Esse levantamento mostrou que foram aplicados 713.000 litros de herbicidas, 96.000 litros de inseticidas e $567.500 \mathrm{~kg}$ de fungicidas na região. A maior quantidade de herbicidas foi aplicada na cultura do milho, seguida pela cultura de oleaginosas.

Estudos de percepção, realizados no Alto Vale do Itajaí, indicaram a preocupação das pessoas com os riscos ambientais associados ao uso de pesticidas na agricultura (BRUGNEROTTO, 2003). No entanto, poucos estudos científicos têm sido desenvolvidos visando identificar as características das moléculas empregadas, bem como a potencialidade de contaminação das águas superficiais e/ou subterrâneas na bacia do rio Itajaí.

Indicadores de risco têm sido apresentados e aplicados na avaliação do potencial de degradação do meio ambiente e da saúde humana (SPADOTTO et al., 2001; ZAHM, 2003; DEVILLERS et al., 2005; SAMUEL et al., 2007). A avaliação de risco consiste na caracterização do potencial adverso dos efeitos da exposição humana a agentes ou atividades perigosas. O uso de métodos de análises matemáticas do risco fornece subsídios objetivos e racionais para realização de diagnósticos e auxílio na tomada de decisão (BRILHANTE e CALDAS, 2002).

Dentre os métodos de avaliação do risco ambiental tem-se o Sistema de Integração de Risco com Interação de Pontuação (Scores) - SIRIS (VAILLANT, JOUANY e DEVILLERS, 1995). Trata-se de método simplificado de avaliação de risco que combina dados a respeito do perigo potencial e da exposição, expressos mediante parâmetros ecotoxicológicos e físico-químicos. Esses fatores são classificados em ordem hierárquica, sendo o efeito e a exposição estimados mediante sistema de pontuações. O método SIRIS não é baseado em dados de monitoramento ou modelagem, mas em dados referenciais. Tratando-se de método de seleção, seu objetivo é classificar substâncias químicas em termos da periculosidade que representam para identificar aquelas que devem ser monitoradas no ambiente de modo prioritário (GUERBET e JOUANY, 2002). IRACE-GUIGAND et al. (2004) verificaram boa concordância entre os resultados obtidos com o método SIRIS e análises de amostras de água coletadas numa bacia hidrográfica.

O risco considerado é apresentado em duas dimensões, ou seja, a probabilidade da exposição e a gravidade das conseqüências (efeitos). Assim, a importância relativa dos critérios torna-se mais facilmente comparável entre duas categorias mais homogêneas. Constrói-se uma escala de classificação baseada no critério de exposição e outra escala levando em conta os efeitos biológicos. Cada 
substância química é inserida como um ponto único no gráfico, com cada um dos aspectos numa das coordenadas, podendo-se analisar a posição da substância química testada em relação às outras e, também, em relação às melhores e piores situações (VAILLANT, JOAUNY e DEVILLERS, 1995).

Este trabalho teve por objetivo estimar o risco de degradação das águas superficiais e subterrâneas, ocasionado pela utilização de pesticidas pelos produtores rurais. Usou-se o método SIRIS para avaliar a bacia do rio Itajaí, região do Alto Vale do Itajaí, situada na parte leste do estado de Santa Catarina. A Bacia do Itajaí é ocupada por 47 municípios com aproximadamente $15.500 \mathrm{~km}^{2}$ (16,15\% do território catarinense).

\section{MATERIAL E METODOS}

O estudo envolveu o levantamento das culturas desenvolvidas na região e suas respectivas áreas, dos produtos comerciais utilizados e suas características, bem como a avaliação de risco de degradação das águas superficiais e subterrâneas da área. A região do Alto Vale do Itajaí considerada (Figura 1) compreende os municípios de Agrolândia, Agronômica, Alfredo Wagner, Atalanta, Aurora, Braço do Trombudo, Imbuia, Ituporanga, Lontras, Petrolândia, Pouso Redondo, Rio do Sul e Trombudo Central. Tais municípios foram escolhidos por estarem situados em região com elevado uso agrícola, cuja área de captação contribui para a reposição de aqüíferos subterrâneos e corpos de água superficiais.

Desenvolveu-se questionário para coleta de dados por cultivos, produtos comerciais e/ou ingredientes ativos utilizados, extensão dos plantios, dose aplicada por superfície, período e modo de aplicação.

As áreas das principais culturas da região do estudo foram determinadas a partir do levantamento agropecuário, realizado em entrevistas com os responsáveis técnicos dos respectivos municípios. Os dados também foram obtidos com base no levantamento agropecuário 2002-2003, disponibilizado pelo Centro de Socioeconomia e Planejamento Agrícola (SANTA CATARINA, 2005).

\section{FIGURA 1 - MUNICÍPIOS DA REGIÃO DO ALTO VALE DO ITAJAÍ QUE COMPREENDEM A ÁREA DE ESTUDO}

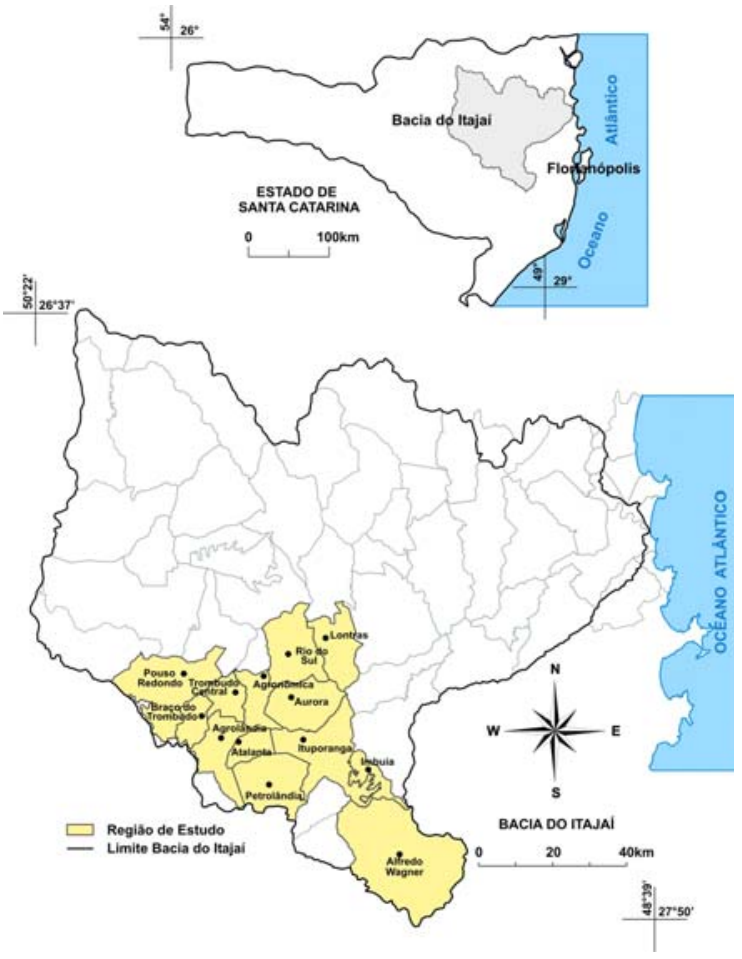


Para obter os dados sobre os ingredientes ativos, utilizados na região do Alto Vale do Itajaí, foi realizado levantamento junto aos serviços de assistência técnica da EPAGRI, CIDASC, cooperativa da CRAVIL, agropecuárias e prefeituras dos municípios em questão. Em geral, considerou-se em cada município apenas um responsável pela informação.

Os ingredientes ativos foram separados quanto a sua ação sobre os organismos vivos em herbicidas, inseticidas e fungicidas, sendo os dados inseridos em planilhas para o tratamento estatístico dos mesmos.

A quantidade real de pesticidas utilizada pelos agricultores varia de ano para ano, dependendo do tipo de praga que ataca a lavoura no período em questão. Não existem registros disponíveis sobre a quantidade de pesticida utilizada por agricultor separadamente, sendo esse valor estimado a partir da quantidade recomendada de aplicação do produto comercial. A dose recomendada foi obtida no banco de dados Sistema de Agrotóxicos Fitossanitários (AGROFIT) do Ministério da Agricultura, Pecuária e Abastecimento.

A composição do produto comercial determina a dose recomendada do ingrediente ativo pelos fabricantes dos produtos formulados e essa varia em função de vários fatores, mas principalmente em razão das pragas a combater (abrangendo de gramas a quilogramas por hectare). As doses recomendadas são consideradas na aplicação do método SIRIS.

A determinação dos ingredientes ativos utilizados nos cultivos, as quantidades efetivamente aplicadas e as datas dessas aplicações variam fortemente no tempo e no espaço. Assim, supõe-se que as doses recomendadas sejam adotadas pelos produtores agrícolas e que todos os ingredientes ativos estejam sendo utilizados de maneira uniforme.

Para cada ingrediente ativo foram pesquisadas as características físicas, químicas e ecotoxicológicas nos bancos de dados especializados AGRITOX (AFSSA, 2007), EXTOXNET (UNIVERSITY OF CALIFORNIA-DAVIS 2007), FOOTPRINT (UNIVERSITY OF HERTFORDSHIRE, 2007), Crop System (ARS-USDA, 2007) e nas bibliografias Pesticide properties in the environment (HORNSBY, WAUCHOPE e HERNEST, 1996), Metabolic athways of agrochemicals (ROBERTS e HUTSON, 1999a e 1999b) e Agrochemicals desk reference (MONTGOMERY, 1997). Levantaramse as seguintes propriedades: solubilidade da água, tempo de meia-vida em campo, tempo de meia-vida de hidrólise e de fotólise, coeficiente de sorção do solo, pressão de vapor, constante de ionização ácido-base, concentração letal em peixes, concentração efetiva em Daphnia magna, concentração efetiva em algas, dose letal via oral em ratos, coeficiente de partição octanol-água, dose diária aceitável, coeficiente de Henry, concentração de predição sem efeito e fator de bioconcentração.

A composição dos produtos comerciais e as doses recomendadas dos ingredientes ativos foram obtidas nas bases de dados AGROFIT (MAPA, 2007) e Sistema de Informações sobre Agrotóxicos SIA (ANVISA, 2007).

O risco é considerado como variável de duas dimensões. Uma dimensão envolve a exposição a que o ambiente está submetido e o outro o efeito que a substância ocasiona no ambiente. Para águas superficiais são considerados os efeitos ecotoxicológicos aos peixes, aos invertebrados aquáticos e às algas, bem como a dose máxima aceitável ao homem. No caso das águas subterrâneas é considerada apenas a dose máxima aceitável ao homem.

Para avaliar as propriedades que devem apresentar maior peso na geração do risco de ocorrência de degradação das massas de água foram estabelecidos intervalos em três níveis: favorável (índice f), intermediário (índice m) e desfavorável (índice d). Os Quadros 1 e 2 fornecem os valores das propriedades consideradas e os respectivos níveis para águas subterrâneas e superficiais, respectivamente.

Para cada ingrediente ativo foram determinados os níveis de exposição em função de suas características específicas e somados os níveis de exposição por características para se obter o valor global do nível de exposição. A soma desses níveis foi realizada com base na escala de peso das propriedades apresentada por DE LAVAUR et al. (1995). Somente os níveis para os valores de superfície de aplicação foram adaptados à realidade da área em estudo, adotando-se o nível $f$ para superfícies 
menores que 2.500 ha, o nível $m$ para superfícies com valores entre 2.500 e 10.000 ha e o nível $d$ para superfícies maiores que 10.000 ha.

\section{QUADRO 1 - CRITÉRIOS UTILIZADOS PARA CLASSIFICAÇÃO DAS PROPRIEDADES FÍSICO- QUÍMICAS PELO MÉTODO SIRIS PARA ÁGUAS SUBTERRÂNEAS}

\begin{tabular}{|c|c|c|c|}
\hline \multicolumn{4}{|c|}{ Classes de Critérios para Águas Subterrâneas } \\
\hline Nivel de Class ific açäo & $\mathbf{f}$ & $\mathbf{m}$ & d \\
\hline Coeficiente de Sorçẫo $-K_{o c}\left(\mathrm{~mL} \mathrm{~g}^{-1}\right)$ & $>500$ & $500-100$ & $<100$ \\
\hline Tempo de Degradaçẫo - DT ${ }_{50}$ (dias) & $<30$ & $30-120$ & $>120$ \\
\hline Hidrólise (dias) & $<30$ & $30-60$ & $>60$ \\
\hline Superfície (ha) & $<2.500$ & $2.500-10.000$ & $>10.000$ \\
\hline Quantidade $\left(\mathrm{kg} \mathrm{ha}^{-1}\right)$ & $\angle 0,5$ & $0,5-1$ & $>1$ \\
\hline Hidrossolubilidade $\left(\mathrm{mg} \mathrm{L}^{-1}\right)$ & $<10$ & $10-200$ & $>200$ \\
\hline
\end{tabular}

* Os valores referentes à superfície de aplicação foram adaptados à realidade da área de estudo em questão. $\mathrm{F}=$ favorável, $\mathrm{m}$ = intermediário, $\mathrm{d}$ = desfavorável.

Fonte: DE LAVAUR et al. (1995).

\section{QUADRO 2 - CRITÉRIOS UTILIZADOS PARA CLASSIFICAÇÃO DAS PROPRIEDADES FÍSICO- QUÍMICAS PELO MÉTODO SIRIS PARA ÁGUAS SUPERFICIAIS}

\begin{tabular}{|c|c|c|c|}
\hline \multicolumn{4}{|c|}{ Class es de Critérios para Águas Superficiais } \\
\hline Nivel de Class ific açäo & $\mathbf{f}$ & $\mathbf{m}$ & d \\
\hline Coeficiente de Sorção $-K_{o c}\left(\mathrm{~mL} \mathrm{~g}^{-1}\right)$ & $>1.000$ & $1.000-100$ & $<100$ \\
\hline Tempo de Degradaçẫo - DT ${ }_{50}$ (dias) & $<8$ & $8-30$ & $>30$ \\
\hline Hidrólise (dias) & $<30$ & $30-60$ & $>60$ \\
\hline Superfície (ha) & $<2.500$ & $2.500-10.000$ & $>10.000$ \\
\hline Quantidade ( $\left.\mathrm{kg} \mathrm{ha}^{-1}\right)$ & $<0,5$ & $0,5-1$ & $>1$ \\
\hline Hidrossolubilidade ( $\mathrm{mg} \mathrm{L}^{-1}$ ) & $<10$ & $10-200$ & $>200$ \\
\hline
\end{tabular}

* Os valores referentes à superfície de aplicação foram adaptados à realidade da área de estudo em questão. $\mathrm{F}$ = favorável, $\mathrm{m}$ = intermediário, $\mathrm{d}$ = desfavorável.

Fonte: DE LAVAUR et al. (1995).

O método SIRIS apresenta os riscos de degradação das águas superficiais e das águas subterrâneas sob forma gráfica, sendo elaborados gráficos individuais para cada massa de água. No gráfico de águas superficiais coloca-se no eixo y a escala de exposição, considerando os valores da exposição obtidos pelas variáveis superfície (ha), quantidade $\left(\mathrm{kg} \mathrm{ha}^{-1}\right)$, solubilidade em água $\left(\mathrm{mg} \mathrm{L}^{-1}\right)$, hidrólise (d), $\mathrm{DT}_{50}$ (d) e Koc $\left(\mathrm{g} \mathrm{mL}^{-1}\right)$. Coloca-se no eixo $\mathrm{x}$, o menor valor do efeito toxicológico em peixes $\left(\mathrm{CL}_{50}\right)$, em daphnia $\left(\mathrm{CE}_{50}\right)$ e em algas $\left(\mathrm{CE}_{50}\right)$, ou a dose diária aceitável ao homem (IDA). Para as águas subterrâneas, coloca-se no eixo $x$ a dose diária aceitável ao homem (IDA) e no y a escala de exposição, obtida a partir de $\mathrm{Koc}_{\mathrm{N}} \mathrm{DT}_{50}$, hidrólise, superfície, quantidade e solubilidade em água.

O risco de degradação das águas subterrâneas e superficiais é crescente. Passa-se do valor de risco mínimo para baixos níveis de exposição e concentrações elevadas com efeitos sobre os organismos aquáticos e/ou ao homem ao valor de risco máximo para exposição e efeitos elevados em baixas concentrações. Os ingredientes ativos com maior risco de degradação das águas situam-se nos extremos superiores dos eixos $x$ e y. $\mathrm{Na}$ interseção entre os dois eixos encontra-se o risco 
mínimo, ou o valor ideal para o qual a substância mostrará nível favorável (f) para todas as propriedades consideradas.

$\mathrm{Na}$ análise da importância do risco para águas superficiais e subterrâneas, os gráficos obtidos foram divididos em 5 níveis nas faixas apresentadas no Quadro 3 (efeitos sobre o homem) e Quadro 4 (efeito sobre os organismos aquáticos). Aplicando esses níveis de risco efetua-se a hierarquização dos ingredientes ativos.

\section{QUADRO 3 - RISCOS DE DEGRADAÇÃO DAS ÁGUAS EM FUNÇÃO DO EFEITO SOBRE O HOMEM}

\begin{tabular}{|c|c|c|}
\hline thin & Prod:hn & 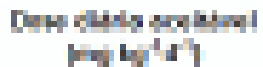 \\
\hline 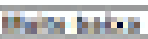 & magnath & handen \\
\hline Artat & 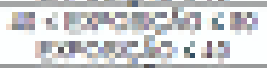 & Tristat \\
\hline Hes & 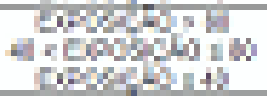 & a migh ind \\
\hline Hit & 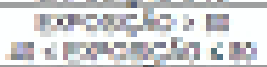 & 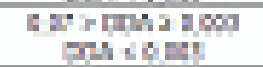 \\
\hline mitrie in & natran & Dy-dis: \\
\hline
\end{tabular}

\section{QUADRO 4 - RISCOS DE DEGRADAÇÃO DAS ÁGUAS EM FUNÇÃO DO EFEITO SOBRE O MEIO AQUÁTICO}

\begin{tabular}{|c|c|c|}
\hline thr & Amtrits & 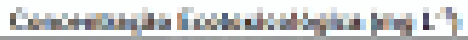 \\
\hline Henten & 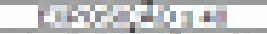 & Mathit \\
\hline mat & 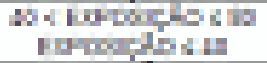 & 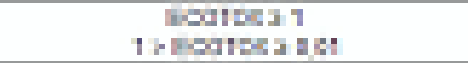 \\
\hline HHA & 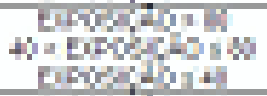 & ingur \\
\hline Ats & 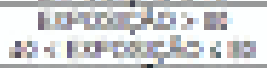 & 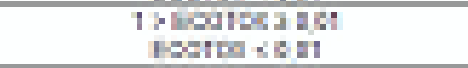 \\
\hline mestes & LHTH, III H & Batm ant \\
\hline
\end{tabular}

\section{RESULTADOS E DISCUSSÃO}

Nas entrevistas com os serviços de assistência técnica foram indicadas como as principais culturas da região: milho, arroz, feijão, fumo, batata, cebola, mandioca, beterraba, abóbora, melancia, tomate e pastagem. Constatou-se que o milho constitui a principal cultura desenvolvida, com uma superfície de cerca de 30 mil ha ao longo dos 13 municípios.

Pelo levantamento dos produtos comerciais utilizados pelos produtores agrícolas na região do Alto Vale do Itajaí foram identificados 39 herbicidas, 33 inseticidas e 32 fungicidas. Observou-se no manejo das diferentes culturas a recomendação do uso de número elevado de pesticidas. Apenas no manejo da cebola tem-se a recomendação de mais de 50 ingredientes ativos diferentes. Situação similar, mas em menor número, são as moléculas recomendadas para o manejo do tomate, milho, batata, fumo, feijão e arroz. Em pastagem é recomendado o uso do herbicida 2,4-D.

O Quadro 5 apresenta os valores de escala de exposição obtidos para os herbicidas, inseticidas e fungicidas utilizados na região do Alto Vale do Itajaí, relacionados com as águas subterrâneas e superficiais. 
Valores de exposição superiores foram observados para as águas superficiais. Isto significa que as águas superficiais estão mais suscetíveis à ação de degradação dos ingredientes ativos utilizados. Os valores máximos de exposição das águas superficiais foram 106, 86 e 77 para herbicidas, inseticidas e fungicidas, respectivamente. Para as águas subterrâneas os valores máximos de exposição foram respectivamente 94,77 e 57 . Os herbicidas, inseticidas e fungicidas apresentaram, respectivamente, valores mínimos de 11, 3 e 6 para águas superficiais e de 3, 0 e 3 para águas subterrâneas.

\section{QUADRO 5 - VALOR DA ESCALA DE EXPOSIÇÃO DOS INGREDIENTES ATIVOS UTILIZADOS NO ALTO VALE DO ITAJAÍ}

\begin{tabular}{|c|c|c|c|c|c|c|c|c|}
\hline \multicolumn{9}{|c|}{ Escala de Exposição } \\
\hline Herbicidas & SUB. & SUP. & Fungicidas & SUB. & SUP. & Inseticidas & SUB. & SUP. \\
\hline $2,4-\mathrm{D}$ & 90 & 96 & Azoxistrobim & 10 & 24 & Abamectina & 0 & 3 \\
\hline Acetocloro & 49 & 78 & Benomil & 21 & 36 & Acefato & 77 & 76 \\
\hline Alacloro & 34 & 57 & Captana & 48 & 54 & $\begin{array}{c}\text { Alfa- } \\
\text { cipermetrina }\end{array}$ & 18 & 14 \\
\hline Atrazina & 92 & 105 & Carbendazim & 43 & 35 & Beta-ciflutrina & 21 & 42 \\
\hline Bentazona & 77 & 86 & Carboxina & 14 & 6 & Carbofurano & 77 & 86 \\
\hline Cletodim & 80 & 66 & Cimoxanil & 25 & 22 & Ciflutrina & 18 & 14 \\
\hline Clomazona & 53 & 67 & Clorotalonil & 18 & 50 & Cipermetrina & 28 & 49 \\
\hline Diurom & 42 & 106 & Difenoconazol & 26 & 33 & Ciromazina & 2 & 12 \\
\hline $\begin{array}{c}\text { Fenoxaprope-p- } \\
\text { etílico }\end{array}$ & 14 & 36 & Famoxadona & 3 & 16 & Clorpirifós & 21 & 42 \\
\hline $\begin{array}{l}\text { Fluazifope-p- } \\
\text { butílico }\end{array}$ & 16 & 26 & Fenamidona & 35 & 30 & Deltametrina & 14 & 36 \\
\hline Flumioxazina & 3 & 13 & Folpete & 17 & 19 & $\begin{array}{c}\text { Diflubenzuro } \\
\mathrm{m}\end{array}$ & 12 & 7 \\
\hline Fomesafem & 71 & 36 & Iprodiona & 43 & 67 & Dimetoato & 69 & 45 \\
\hline Foramsulfurom & 94 & 59 & Iprovalicarbe & 37 & 50 & Fenitrotiom & 47 & 58 \\
\hline Glifosato & 29 & 89 & Mancozebe & 14 & 55 & Fipronil & 16 & 30 \\
\hline Iodossulfurom & 69 & 46 & Metalaxil-M & 35 & 76 & Imidaclopride & 65 & 53 \\
\hline Ioxinil & 57 & 86 & Metconazol & 32 & 39 & Indoxacarbe & 6 & 7 \\
\hline Isoxaflutol & 17 & 13 & Metiram & 10 & 39 & $\begin{array}{l}\text { Lambda- } \\
\text { cialotrina }\end{array}$ & 7 & 29 \\
\hline Mesotriona & 37 & 23 & Piraclostrobina & 14 & 36 & Lufenurom & 16 & 26 \\
\hline Metribuzim & 59 & 30 & Pirimetanil & 47 & 65 & Metamidofós & 54 & 49 \\
\hline $\begin{array}{l}\text { Metsulfurom- } \\
\text { metílico }\end{array}$ & 57 & 45 & Procimidona & 18 & 50 & Metiocarbe & 40 & 37 \\
\hline Nicossulfurom & 55 & 64 & Procloraz & 31 & 58 & Novalurom & 18 & 14 \\
\hline Oxadiazona & 18 & 50 & Propiconazol & 26 & 33 & $\begin{array}{l}\text { Parationa- } \\
\text { metílica }\end{array}$ & 27 & 43 \\
\hline Oxifluorfem & 3 & 11 & Propinebe & 57 & 60 & Profenofós & 4 & 17 \\
\hline Paraquate & 36 & 48 & Tebuconazol & 39 & 59 & Tiametoxam & 69 & 45 \\
\hline Pendimentalina & 18 & 50 & Tiabendazol & 44 & 77 & Tiodicarbe & 37 & 50 \\
\hline Picloram & 93 & 83 & Tiofanato-metílico & 12 & 22 & Triclorfom & 54 & 57 \\
\hline Pirazossulfurom & 4 & 25 & Tiram & 12 & 57 & Triflumurom & 12 & 10 \\
\hline Quincloraque & 66 & 40 & Trifloxistrobina & 21 & 36 & & & \\
\hline $\begin{array}{c}\text { Quizalofope-p- } \\
\text { etílico }\end{array}$ & 14 & 24 & & & & & & \\
\hline Setoxidim & 69 & 45 & & & & & & \\
\hline Simazina & 58 & 73 & & & & & & \\
\hline S-Metolacloro & 24 & 62 & & & & & & \\
\hline
\end{tabular}

SUB = água subterrânea; SUP = água superficial. 
Os valores máximos de exposição para águas superficiais e subterrâneas foram determinados para os mesmos ingredientes ativos. Os herbicidas que obtiveram valores máximos na escala de exposição foram o Diurom para águas superficiais e o Foramsulfurom para águas subterrâneas. Dentre os inseticidas, o Carbofurano mostrou o maior valor de exposição nos dois tipos de massa de água. Os fungicidas que obtiveram maiores valores para escala de exposição foram o Tiabendazol para águas superficiais e o Propinebe para águas subterrâneas.

No caso de valores mínimos, o fungicida Famoxadona, os herbicidas Oxifluorfem e Flumioxazina, e o inseticida Abamectina apresentaram valores baixos na escala de exposição tanto para águas superficiais quanto para águas subterrâneas.

As Figuras 2 a 4 apresentam os gráficos de riscos de degradação das águas superficiais e das águas subterrâneas, estando no eixo x os efeitos ao homem ou aos organismos aquáticos e no eixo y os valores da escala de exposição. No eixo $x$, os valores de ingestão diária aceitável (IDA) ao homem variaram de 0,0001 a $1 \mathrm{mg} \mathrm{kg}^{-1} \mathrm{~d}^{-1}$ e o efeito ecotoxicológico de 0,0001 a $100 \mathrm{mg} \mathrm{L}^{-1}$.

Os inseticidas situaram-se, principalmente, entre as faixas de risco intermediário a risco máximo de degradação das águas superficiais e subterrâneas. Os fungicidas ficaram distribuídos nas faixas intermediárias de risco. Já os herbicidas, distribuídos por todo o gráfico, evidenciaram ingredientes ativos próximos de riscos mínimos e máximos.

\section{FIGURA 2 - RISCO DE DEGRADAÇÃO DAS ÁGUAS SUPERFICIAIS PARA O HOMEM}

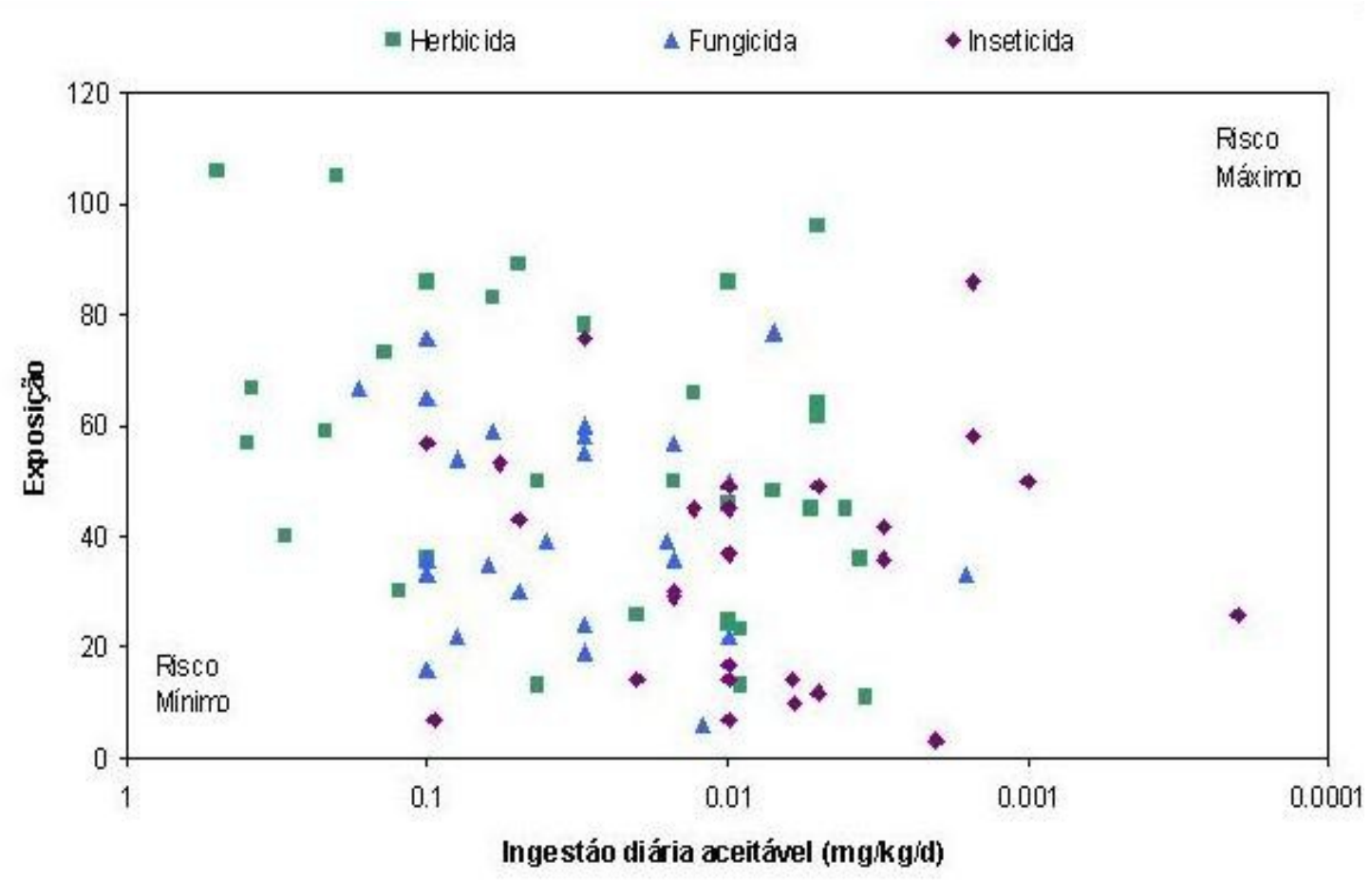

O Quadro 6 apresenta a hierarquização dos riscos de degradação das águas superficiais por pesticidas no Alto Vale do Itajaí e o Quadro 7 para águas subterrâneas. Verificou-se em relação ao efeito sobre o homem que cerca de $68 \%$ dos fungicidas, $38 \%$ dos herbicidas e $44 \%$ dos inseticidas apresentaram risco baixo ou muito baixo para águas superficiais. Para águas subterrâneas, esses valores abrangeram cerca de $86 \%$ dos fungicidas, $60 \%$ dos herbicidas e $66 \%$ dos inseticidas. Ainda para o efeito sobre o homem, cerca de $4 \%$ dos fungicidas, $19 \%$ dos herbicidas e $22 \%$ dos inseticidas apresentaram risco alto ou muito alto para águas superficiais e $6 \%$ dos herbicidas e $11 \%$ inseticidas 
para águas subterrâneas. Dentre os fungicidas não foi encontrado nenhum ingrediente ativo classificado como risco alto ou muito alto para águas subterrâneas.

\section{FIGURA 3 - RISCO DE DEGRADAÇÃO DAS ÁGUAS SUPERFICIAIS PARA OS ORGANISMOS AQUÁTICOS}

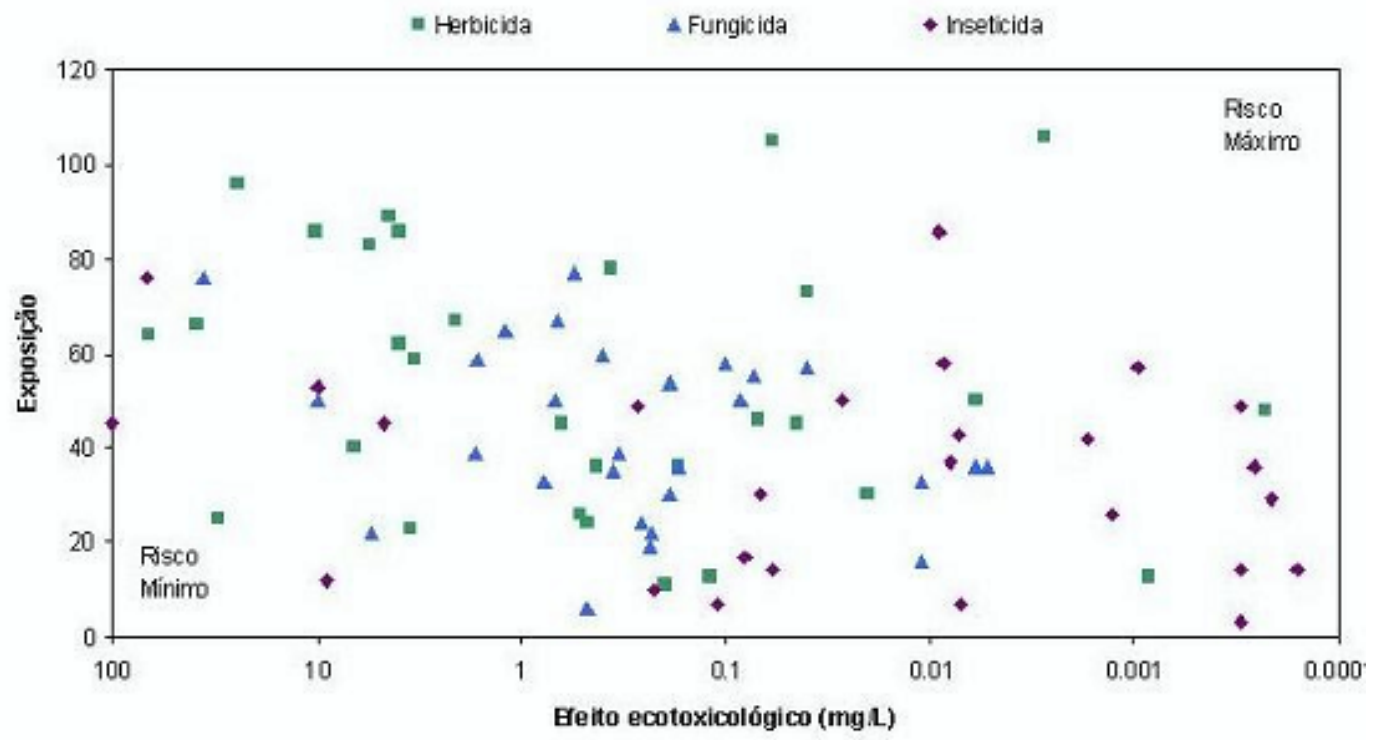

FIGURA 4 - RISCO DE DEGRADAÇÃO DAS ÁGUAS SUBTERRÂNEAS

PARA O HOMEM

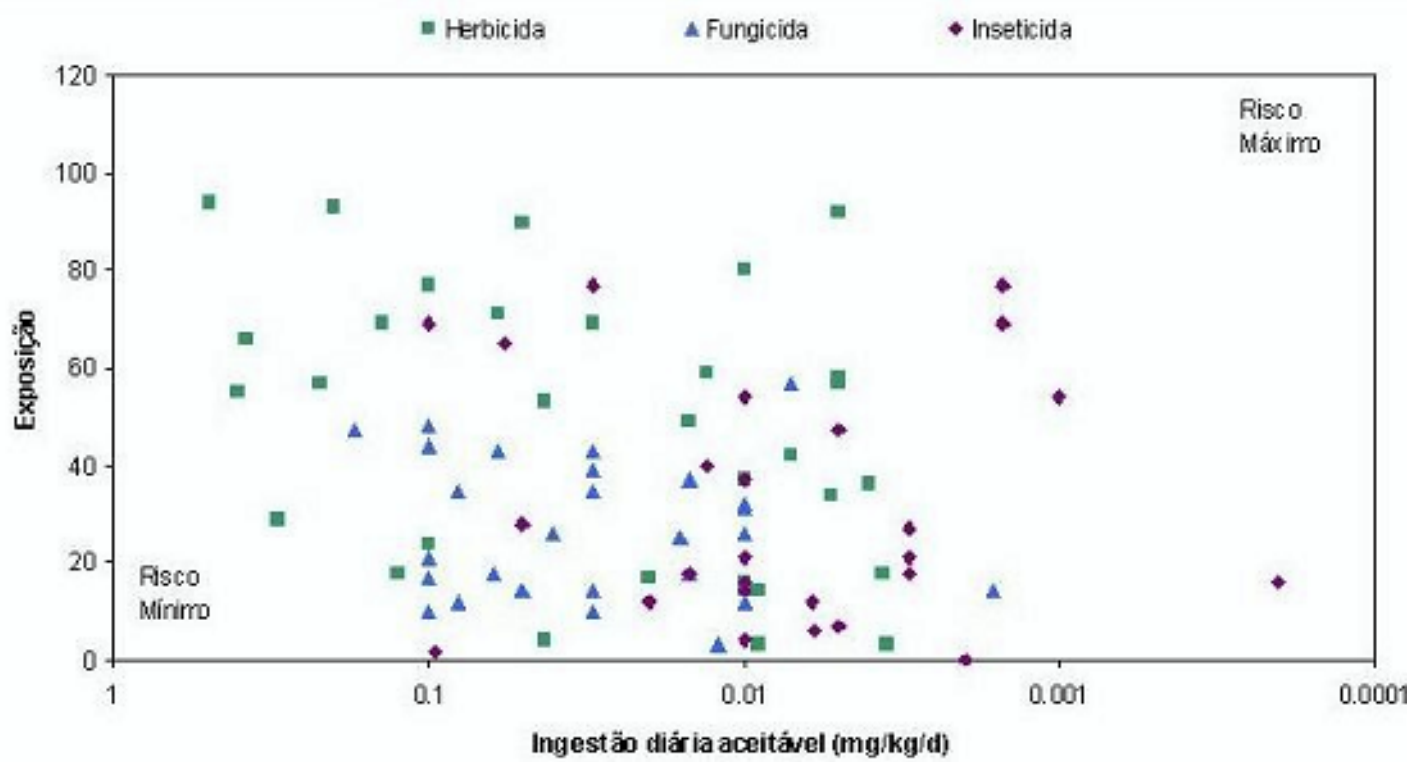




\begin{tabular}{|c|c|c|c|c|c|c|c|}
\hline \multicolumn{8}{|c|}{ Efeitos sobre o homem } \\
\hline Risco & Herbicida & Fungicida & Inseticida & Risco & Herbicida & Fungicida & Inseticida \\
\hline $\begin{array}{l}\text { Muito } \\
\text { baixo }\end{array}$ & $\begin{array}{l}\text { S-Metolacloro } \\
\text { Glifosato } \\
\text { Pendimentalina }\end{array}$ & $\begin{array}{l}\text { Azoxistrobim } \\
\text { Benomil } \\
\text { Folpete } \\
\text { Metalaxil-M } \\
\text { Tiofanato-metílico } \\
\text { Trifloxistrobina }\end{array}$ & Ciromazina & Médio & $\begin{array}{l}\text { Acetocloro } \\
\text { Cletodim } \\
\text { Clomazona } \\
\text { Fomesafem } \\
\text { Foramsulfurom } \\
\text { lodossulfurom } \\
\text { loxinil } \\
\text { Metribuzim } \\
\text { Picloram } \\
\text { Simazina } \\
\text { Diurom }\end{array}$ & $\begin{array}{l}\text { Carbendazim } \\
\text { Carboxina } \\
\text { Propinebe } \\
\text { Iprodiona }\end{array}$ & $\begin{array}{l}\text { Abamectina } \\
\text { Acefato } \\
\text { Beta-ciflutrina } \\
\text { Ciflutrina } \\
\text { Fenitrotiom } \\
\text { Fipronil } \\
\text { Imidaclopride } \\
\text { Parationa- } \\
\text { metílica } \\
\text { Triclorfom }\end{array}$ \\
\hline Baixo & $\begin{array}{l}\text { Alacloro } \\
\text { Bentazona } \\
\text { Fenoxaprope-p- } \\
\text { etílico } \\
\text { Fluazifope-p- } \\
\text { butílico } \\
\text { Flumioxazina } \\
\text { Isoxaflutol } \\
\text { Mesotriona } \\
\text { Metsulfurom- } \\
\text { metílico } \\
\text { Nicossulfurom } \\
\text { Oxadiazona } \\
\text { Oxifluorfem } \\
\text { Paraquate } \\
\text { Pirazossulfurom } \\
\text { Quincloraque } \\
\text { Quizalofope-p- } \\
\text { etílico } \\
\text { Setoxidim }\end{array}$ & $\begin{array}{l}\text { Captana } \\
\text { Cimoxanil } \\
\text { Clorotalonil } \\
\text { Difenoconazol } \\
\text { Famoxadona } \\
\text { Fenamidona } \\
\text { Iprovalicarbe } \\
\text { Mancozebe } \\
\text { Metconazol } \\
\text { Metiram } \\
\text { Piraclostrobina } \\
\text { Pirimetanil } \\
\text { Procimidona } \\
\text { Procloraz } \\
\text { Propiconazol } \\
\text { Tebuconazol } \\
\text { Tiabendazol } \\
\text { Tiram }\end{array}$ & $\begin{array}{l}\text { Alfa-Cipermetrina } \\
\text { Cipermetrina } \\
\text { Clorpirifós } \\
\text { Deltametrina } \\
\text { Diflubenzurom } \\
\text { Indoxacarbe } \\
\text { Lambda-cialotrina } \\
\text { Lufenurom } \\
\text { Metiocarbe } \\
\text { Novalurom } \\
\text { Profenofós } \\
\text { Tiametoxam } \\
\text { Tiodicarbe } \\
\text { Triflumurom }\end{array}$ & Alto & $\begin{array}{l}2,4-\mathrm{D} \\
\text { Atrazina }\end{array}$ & & $\begin{array}{l}\text { Carbofurano } \\
\text { Dimetoato } \\
\text { Metamidofós }\end{array}$ \\
\hline
\end{tabular}


QUADRO 7 - HIERARQUIZAÇÃO DOS RISCOS DE DEGRADAÇÃO POR PESTICIDAS

DAS ÁGUAS SUPERFICIAIS NO ALTO VALE DO ITAJAÍ

\begin{tabular}{|c|c|c|c|c|c|c|}
\hline & \multicolumn{3}{|c|}{ Efeito sobre o homem } & \multicolumn{3}{|c|}{ Efeito sobre organismos aquáticos } \\
\hline Risco & Herbicida & Fungicida & Inseticida & Herbicida & Fungicida & Inseticida \\
\hline Muito baixo & $\begin{array}{l}\text { Fenoxaprope-p-etílico } \\
\text { Fluazifope-p-butílico } \\
\text { Metribuzim } \\
\text { Oxifluorfem } \\
\text { Quizalofope-p-etílico } \\
\text { Pirazossulfurom } \\
\text { Mesotriona }\end{array}$ & $\begin{array}{l}\text { Piraclostrobina } \\
\text { Cimoxanil } \\
\text { Propiconazol } \\
\text { Metconazol } \\
\text { Tiofanato-metílico }\end{array}$ & $\begin{array}{l}\text { Profenofós } \\
\text { Ciromazina }\end{array}$ & $\begin{array}{l}\text { Pirazossulfurom } \\
\text { Mesotriona } \\
\text { Quincloraque }\end{array}$ & $\begin{array}{l}\text { Metconazol } \\
\text { Tiofanato-metílico }\end{array}$ & Ciromazina \\
\hline Baixo & $\begin{array}{l}\text { Flumioxazina } \\
\text { lodosulfurom } \\
\text { Simazina } \\
\text { Fomesafem } \\
\text { Isoxaflutol }\end{array}$ & $\begin{array}{l}\text { Trifloxistrobina } \\
\text { Azoxistrobim } \\
\text { Benomil } \\
\text { Carbendazim } \\
\text { Carboxina } \\
\text { Difenoconazol } \\
\text { Famoxadona } \\
\text { Fenamidona } \\
\text { Folpete } \\
\text { Metalaxil-M } \\
\text { Metiram } \\
\text { Pirimetanil } \\
\text { Iprovalicarbe } \\
\text { Tebuconazol }\end{array}$ & $\begin{array}{l}\text { Abamectina } \\
\text { Ciflutrina } \\
\text { Diflubenzurom } \\
\text { Lambda-cialotrina } \\
\text { Lufenurom } \\
\text { Metiocarbe } \\
\text { Fipronil } \\
\text { Indoxacarbe } \\
\text { Novalurom } \\
\text { Triflumurom }\end{array}$ & $\begin{array}{l}\text { S-Metolacloro } \\
\text { Cletodim } \\
\text { Clomazona } \\
\text { Fenoxaprope-p-etílico } \\
\text { Fluazifope-p-butílico } \\
\text { Fomesafem } \\
\text { Foramsulfurom } \\
\text { Isoxaflutol } \\
\text { Metribuzim } \\
\text { Nicosulfurom } \\
\text { Oxifluorfem } \\
\text { Quizalofope-p-etílico }\end{array}$ & $\begin{array}{l}\text { Azoxistrobim } \\
\text { Benomil } \\
\text { Carbendazim } \\
\text { Carboxina } \\
\text { Cimoxanil } \\
\text { Difenoconazol } \\
\text { Famoxadona } \\
\text { Fenamidona } \\
\text { Folpete } \\
\text { Metalaxil-M } \\
\text { Metiram } \\
\text { Pirimetanil } \\
\text { Propiconazol } \\
\text { Iprovalicarbe } \\
\text { Tebuconazol }\end{array}$ & $\begin{array}{l}\text { Acefato } \\
\text { Dimetoato } \\
\text { Fipronil } \\
\text { Imidaclopride } \\
\text { Indoxacarbe } \\
\text { Novalurom } \\
\text { Profenofós } \\
\text { Triflumurom } \\
\text { Tiametoxam }\end{array}$ \\
\hline Médio & $\begin{array}{l}\text { Alacloro } \\
\text { Paraquate } \\
\text { Pendimentalina } \\
\text { Oxadiazona } \\
2,4-D \\
\text { Acetocloro } \\
\text { Metsulfurom-metílico } \\
\text { Setoxidim } \\
\text { S-Metolacloro } \\
\text { Cletodim } \\
\text { Clomazona } \\
\text { Foramsulfurom } \\
\text { Nicosulfurom } \\
\text { Quincloraque }\end{array}$ & $\begin{array}{l}\text { Captana } \\
\text { Iprodiona } \\
\text { Mancozebe } \\
\text { Procimidona } \\
\text { Procloraz } \\
\text { Propinebe } \\
\text { Tiabendazol } \\
\text { Tiram }\end{array}$ & $\begin{array}{l}\text { Triclorfom } \\
\text { Alfa-cipermetrina } \\
\text { Deltametrina } \\
\text { Metamidofós } \\
\text { Tiodicarbe } \\
\text { Acefato } \\
\text { Dimetoato } \\
\text { Imidaclopride } \\
\text { Tiametoxam }\end{array}$ & $\begin{array}{l}2,4-D \\
\text { Acetocloro } \\
\text { Flumioxazina } \\
\text { Glifosato } \\
\text { lodossulfurom } \\
\text { Metsulfurom-metílico } \\
\text { Picloram } \\
\text { Setoxidim } \\
\text { Simazina } \\
\text { Bentazona } \\
\text { loxinil }\end{array}$ & $\begin{array}{l}\text { Captana } \\
\text { Clorotalonil } \\
\text { Iprodiona } \\
\text { Mancozebe } \\
\text { Piraclostrobina } \\
\text { Procimidona } \\
\text { Procloraz } \\
\text { Propinebe } \\
\text { Tiabendazol } \\
\text { Tiram } \\
\text { Trifloxistrobina }\end{array}$ & $\begin{array}{l}\text { Abamectina } \\
\text { Alfa-cipermetrina } \\
\text { Ciflutrina } \\
\text { Deltametrina } \\
\text { Diflubenzurom } \\
\text { Lambda-cialo trina } \\
\text { Lufenurom } \\
\text { Metamidofós } \\
\text { Metiocarbe } \\
\text { Tiodicarbe }\end{array}$ \\
\hline Alto & $\begin{array}{l}\text { Diurom } \\
\text { Atrazina } \\
\text { Glifosato } \\
\text { Picloram } \\
\text { Bentazona } \\
\text { loxinil } \\
\end{array}$ & Clorotalonil & $\begin{array}{l}\text { Cipermetrina } \\
\text { Clorpirifós } \\
\text { Fenitrotiom } \\
\text { Beta-ciflutrina } \\
\text { Parationa-metílica }\end{array}$ & $\begin{array}{l}\text { Atrazina } \\
\text { Alacloro } \\
\text { Paraquate } \\
\text { Pendimentalina } \\
\text { Oxadiazona } \\
\end{array}$ & & $\begin{array}{l}\text { Cipermetrina } \\
\text { Clorpirifós } \\
\text { Fenitrotiom } \\
\text { Triclorfom } \\
\text { Beta-ciflutrina } \\
\text { Parationa-metílica }\end{array}$ \\
\hline Muito alto & & & & Diurom & & Carbofurano \\
\hline
\end{tabular}


Em relação aos organismos aquáticos, $61 \%$ dos fungicidas, $47 \%$ dos herbicidas e $37 \%$ dos inseticidas apresentaram risco baixo ou muito baixo para degradação das águas superficiais. No entanto, $19 \%$ dos herbicidas e $26 \%$ dos inseticidas foram classificados como risco alto ou muito alto. Assim como para águas subterrâneas, dentre os fungicidas não foi encontrado nenhum ingrediente ativo classificado como risco alto ou muito alto de degradação das águas superficiais.

Deve ser ressaltado que somente o inseticida Carbofurano e o herbicida Diurom foram classificados como ingredientes ativos com risco muito alto de degradação das águas superficiais. Paraáguas subterrâneas nenhum dos ingredientes ativos relatados apresentou risco muito alto de degradação.

Os resultados das análises pelo método SIRIS para águas subterrâneas e águas superficiais podem diferir para a mesma substância, devido ao fato de diferentes características serem consideradas mais ou menos importantes nas escalas de pontuação. Para as águas subterrâneas tem-se a seguinte ordem de importância para as características consideradas: $\mathrm{Koc}_{\text {, }} \mathrm{DT}_{50}$ e hidrólise (juntamente), superfície e quantidade de aplicação (juntamente) e solubilidade. No caso das águas superficiais tem-se a seguinte ordem de importância paras as características consideradas: quantidade e superfície de aplicação (juntamente), solubilidade, DT $_{50}$ e hidrólise (juntamente) e Koc. Com o peso das características praticamente invertido para águas superficiais e subterrâneas, dependendo do valor de uma ou várias características poderá ser obtido resultado muito diferente para a mesma substância.

FERRACINI et al. (2001) utilizando os métodos EPA, índice de GUS e o método GOSS afirmaram que os princípios ativos Diurom, Metalaxil e Procloraz atenderam todos os critérios de avaliação estabelecidos, indicando potencial de contaminação de águas subterrâneas. A meia-vida do Acefato, Dimetoato, Metamidofós e Triclorfom no solo não atenderam aos critérios da avaliação, mas as demais propriedades sim, o que os coloca sob suspeita de causarem contaminação de águas subterrâneas. O Tebuconazol, cujo coeficiente de adsorção não atendeu aos critérios da avaliação, também está sob suspeita de causar contaminação de águas subterrâneas. Após a avaliação de risco, os compostos Abamectina, Clorpirifós, Deltametrina, Iprodiona, Mancozebe e Tiram foram considerados como não-contaminantes de águas subterrâneas.

PRIMEL et al. (2005) utilizaram os mesmos métodos empregados por FERRACINI et al. (2001) e encontraram alto potencial de poluição de águas de superfície para o Clomazona e Propanil. O Quincloraque apresentou potencial médio e o 2,4-D baixo potencial em relação à solubilidade. Quanto ao transporte no sedimento em suspensão, os herbicidas Clomazona, 2,4-D e Propanil mostraram baixo potencial de poluição de águas de superfície e Bentazona e Quincloraque potencial médio de poluição dessas águas.

\section{CONCLUSÃO}

As avaliações dos potenciais de degradação das águas superficiais e das águas subterrâneas indicaram que na região do Alto Vale do Itajaí são utilizados cerca de 39 ingredientes ativos de herbicidas, 33 de inseticidas e 32 de fungicidas nos cultivos de milho, cebola, fumo, arroz, feijão e pastagem. Somente na cultura de cebola são utilizados cerca de 50 ingredientes ativos de pesticidas.

Pelo método SIRIS, poucos ingredientes ativos foram classificados no nível de risco alto ou muito alto de degradação das águas superficiais e de águas subterrâneas.

O herbicida Diurom e o inseticida Carbofurano são os ingredientes ativos com risco muito alto de degradação das águas superficiais.

\section{ABSTRACT}

\section{CLASSIFICATION OF THE PESTICIDES USED ON THE ITAJAI RIVER HYDROGRAPHICAL BASIN (SC)} BRAZIL FOR THE RISK OF HIDRIC RESOURCES DEGRADATION

The objective of this work was to estimate the risk of superficial and ground water's degradation on the Itajaí River's Basin, more specifically at the region of Itajaí's High Valley (SANTA CATARINA - BRAZIL), caused by the utilization of pesticides by rural producers. The study evolved a data-collecting of the 
developed cultures and their respective areas, of the chemicals used and their characteristics, and the evaluation of superficial and ground water degradation's risk (SIRIS method) in the highest part of Itajaí River's Basin. It was evidenced around 39 active substances, 33 from insecticides and 32 fungicides are used at corn, onion, tobacco, rice, bean and grass cultures. At the onion culture are used around 50 active ingredients of pesticides. The SIRIS method classified few chemicals as high or very high risk of superficial and ground water's degradation. Otherwise, the herbicide Diuron and the insecticide Carbofuran are the chemicals considered as very high risk of superficial and ground water's degradation.

KEY-WORDS: ENVIRONMENTAL RISK ASSESSING; PESTICIDES; SUPERFICIAL WATER; GROUND WATER; HIGH ITAJAÍ VALLEY.

\section{REFERÊNCIAS}

1 AFSSA. Agence Francaise de Securité Sanitaire des Aliments. AGRITOX: base de données sur les substances actives phytopharmaceutiques. Disponível em: http://www.dive.afssa.fr/agritox/index.php. Acesso em: 29/07/2007.

2 ANVISA. Agência Nacional de Vigilância Sanitária. SIA: sistema de informações sobre agrotóxicos. Disponível em: http://www4.anvisa.gov.br/agrosia/asp/default.asp. Acesso em: 29/07/2007.

3 ARS-USDA. Agricultural Research Service of United States Department of Agriculture. CROP SYSTEM: the ARS pesticide properties database. Disponível em: http://www.ars.usda.gov/services/docs.htm?docid=14199. Acesso em: 29/07/2007.

4 BATTAGLIN, W.A.; THURMAN, E.M.; KALKHOFF S.J.; PORTER, S.D. Herbicides and transformation products in surface waters of the midwestern United States. Journal of the American Water Resources Association, Middleburg, v.39, n.4, p-743-756. 2003.

5 BOWMER, K.H.; KORTH, W.; SCOTT, A.; MCCORKELLE, G.; THOMAS, M. Pesticide monitoring in the irrigation areas of south-western NSW 1990 - 1995. Austrália: CSIRO Land and Water, 1998, 158 p. (Technical Report 17/98).

6 BRILHANTE O.M.; CALDAS, L.Q. (org.). Gestão e avaliação de risco em saúde ambiental. Rio de Janeiro: Editora FIOCRUZ, 2002. $155 \mathrm{p}$.

7 BRUGNEROTTO, O. Avaliação da percepção ambiental sobre agrotóxicos e biotecnologia moderna da população do Alto Vale do Itajaí - SC. Blumenau, 2003. 173 p. Dissertação (Mestrado em Engenharia Ambiental), Fundação Universidade Regional de Blumenau.

8 COUTINHO, C.F.B.; TANIMOTO, S.T.; GALI, A.; GARBELLINI, G.S.; TAKAYAMA, M.; AMARAL, R.B.; MAZO, L.H.; AVACA, L.A.; MACHADO, S.A.S. Pesticidas: mecanismo de ação, degradação e toxidez. Pesticidas: revista de ecotoxicologia e meio ambiente, Curitiba, v.15, p. 65-72, 2005.

9 DE LAVAUR, E. et al. Classements des substances actives phytosanitaires en vue de la surveillance de la qualité des eaux a l'échelle nationale. Paris: Ministère de l'Agriculture et de la Pêche, Ministère de l'Environnement, Ministère Chargé de la Santé, 1995. 51 p.

10 DEVILLERS, J.; FARRET, R.; GIRARDIN, P.; RIVIERE, J-L.; SOULAS, G. Indicateurs pour évaluer les risques liés à l'utilisation des pesticides. Paris: Lavoisier, 2005. 278 p.

11 EPAGRI. Empresa de Pesquisa Agropecuária e Extensão Rural de Santa Catarina. Relatório da Gerência Regional de Rio do Sul. Rio do Sul, 2001. 35 p. (Relatório Interno).

12 FERRACINI, V.L; PESSOA, M.C.Y.P.; SILVA, A.S.; SPADOTTO, C.A. Análise de risco de contaminação das águas subterrâneas e superficiais da região de Petrolina (PE) e Juazeiro (BA). Pesticidas: revista de ecotoxicologia e meio ambiente, Curitiba, v.11, p. 1-16, jan./dez. 2001.

13 GUERBET, M.; JOUANY, J.M. Value of the SIRIS method for the classification of a series of 90 chemicals according to risk for the aquatic environment. Environmental Impact Assessment Review, Amsterdam, v.22, p.377-391, 2002.

14 HORNSBY, A.; WAUCHOPE, R.D.; HERNEST, A. Pesticides properties in the environment. New York: SpringerVerlag, 1996, $227 \mathrm{p}$.

15 IBGE. Instituto Brasileiro de Geografia e Estatística. Pesquisa de Informações básicas municipais: perfil dos municípios brasileiros - meio ambiente 2002. Rio de Janeiro, 2005. 382 p. (Relatório final).

16 IRACE-GUIGAND, S.; AARON, J.J.; SCRIBE, P.; BARCELO, D. A comparison of the environmental impact of pesticide multiresidues and their occurrence in river waters surveyed by liquid chromatography coupled in tandem with UV diode array detection and mass spectrometry. Chemosphere, Amsterdam, v.55, n.7, p.973-981, 2004. 
17 KOLPIN, D.W.; BARBASH J.E.; GILLIOM, R.J. Pesticides in ground water of the United States, 1992-1996. Ground Water, Madison, v.38, p.858-863, 2000.

18 MAPA. Ministério da Agricultura, Pecuária e Abastecimento. AGROFIT: sistema de agrotóxicos fitossanitários. Disponível em: http://extranet.agricultura.gov.br/agrofit_cons/principal_agrofit_cons. Acesso em: 29/07/2007.

19 MONTGOMERY, J.H. Agrochemicals desk reference. $2^{\text {nd }}$ ed. Michigan: Lewis Publishers, 1997. 696 p.

20 PRIMEL, E.G.; ZANELLA, R.; KURZ, M.H.S.; GONÇALVEZ, F.F.; MACHADO, S.O.; MARCHEZAN, E. Poluição das águas por herbicidas utilizados no cultivo do arroz irrigado na região central do estado do Rio Grande do Sul, Brasil: predição teórica e monitoramento. Química Nova, São Paulo, v. 28, n. 4, p. 605-609, 2005.

21 ROBERTS, T.; HUTSON, D. Metabolic pathways of agrochemicals. Part 1: herbicides and plant growth regulators. Cambridge, U.K: The Royal Society of Chemistry,1999a. 849 p.

22 ROBERTS, T.; HUTSON, D. Metabolic pathways of agrochemicals. Part 2: insecticides and fungicides. Cambridge, UK: The Royal Society of Chemistry, 1999b. 1475 p.

23 SAMUEL, O.; DION, S.; ST.LAURENT, L.; APRIL, M-H. Indicateur de risque des pesticides du Québec - IRPeQ santé et environnement. Québec: MAPAQ/MDDEP/INSPD, 2007.52 p.

24 SANTA CATARINA. Secretaria de Estado da Agricultura e Desenvolvimento Rural. Levantamento agropecuário de Santa Catarina: 2002-2003. Florianópolis: EPAGRI, 2005. 255 p.

25 SCHULZ, R. Field studies on exposure, effects, and risk mitigation of aquatic nonpoint-source insecticide pollution: a review. Journal of Environmental Quality, Madison, v.33, p-419-448, 2004.

26 SINDAG. Sindicato Nacional da Indústria de Produtos para Defesa Agrícola. Posicionamento do setor. Defesa Agrícola, n. 3, fev. 2006. Disponível em: http://www.sindag.com.br/ Acesso em: 16/09/2006.

27 SPADOTTO, C.A.; FILIZOLA, H.; GOMES, M.A.F. Avaliação do potencial de lixiviação de pesticidas em latossolo da região de Guaíra, SP. Pesticidas: revista de ecotoxicologia e meio ambiente, Curitiba, v. 11, p.127-136, 2001.

28 UNIVERSITY OF CALIFORNIA-DAVIS. EXTOXNET EXtension TOXicology NETwork. Disponível em: http:// extoxnet.orst.edu/. Acesso em: 29/07/2007.

29 UNIVERSITY OF HERTFORDSHIRE. FOOTPRINT: pesticide properties database. Disponível em: http://sitem.herts.ac.uk/ aeru/footprint/en/index.htm. Acesso em: 29/07/2007.

30 VAILLANT, M.; JOUANY, J.M.; DEVILLERS, J. A multicriteria estimation of the environmental risk of chemicals with the SIRIS method. Toxicology Modeling, Oxford, v. 1, n. 1, p.57-72, 1995.

31 ZAHM, F. Méthodes de diagnostic des exploitations agricoles et indicateurs: panorama et cas particuliers appliqués à l'évaluations des pratiques phytossanitaires. Ingénieries Eau-Agriculture-Territoires, Antony, n.33, p.13-34, 2001.

\section{AGRADECIMENTOS}

Gostaríamos de agradecer ao CNPq, Processo 470736/2005-6 pelo financiamento, a CAPES pela bolsa do segundo autor. 